\title{
LA NO NEUTRALIDAD EN LA EVALUACION DE LA CALIDAD Y MODELOS DE EVALUACION DE LA EDUCACIÓN SUPERIOR, CASOS DE: COLOMBIA, ARGENTINA Y BRASIL ${ }^{1}$
}

\author{
Nina Eleonor Vizcarra Herles* \\ Edwin Guido Boza Condorena** \\ Elisabete Monteiro de Aguiar Pereira*** \\ Recebido: set. 2010 \\ Aprovado: mar. 2011
}
* Doctora en Administración y pos doctoranda en Educación UNICAMP, SP, Brasil. E-mail: herleseleonor@ yahoo.com.br
**Doctor en Administración, Profesor, Universidad Nacional del Altiplano, Puno, Perú. E-mail: ebozac2003@ yahoo.es
***Doctora en Educación, Profesora, Facultad de Educación, UNICAMP, SP, Brasil. E-mail: eaguiar@ unicamp.br

Resumen: En este artículo en base al análisis documental y de la literatura del área se aborda el tema de la no neutralidad de la evaluación de la calidad, se exploran dimensiones en el concepto de calidad proponiendo un modelo comprensivo, se identifican factores que contribuyen a la no neutralidad del concepto en referencia a las instituciones universitarias. Se enfatiza en que los elementos de referencia considerados valiosos para el evaluador (modelo de referencia) comunican características diferenciales a los modelos de evaluación institucional y de programas para educación superior haciendo mención a los casos de Colombia, Argentina y Brasil y se analizan las diversas implicancias en su funcionamiento. Se infiere que contribuyen a la no neutralidad de la evaluación de la calidad: el énfasis conceptual en alguna o algunas dimensiones de la calidad, el propósito que es de interés del evaluador, el cual determina el referente de calidad a utilizar, y las demandas de calidad endógenas y exógenas a la institución, programa o sistema que es objeto de evaluación.

Palabras clave: Evaluación de la calidad; Educación superior; Evaluación institucional; No neutralidad.

\section{NEUTRALITY IN QUALITY EVALUATION AND IN MODELS OF HIGHER EDUCATION EVALUATION - THE CASES OF COLOMBIA, ARGENTINA AND BRAZIL}

Abstract: This article founded on documental analysis as well as on analysis of the literature in the field, addresses the issue of non-neutrality of quality assessment; it discusses dimensions of the quality concept, identifies factors that contribute to the non-neutrality of quality in reference to universities. It emphasizes that the benchmarks that are considered valuable to the evaluator (reference model), give different characteristics to the institutional and program evaluation models for higher education, making reference to the cases of Colombia, Argentina and Brazil and discusses the different implications of its operation. It is inferred that what contributes to the non-neutrality of quality assessment are: the conceptual emphasis on one or on several quality dimensions, the purpose of the evaluator, which determines the quality benchmarks he uses, and the demands for quality which are endogenous or exogenous to the institution, program or system that is under evaluation.

Key words: Quality assessment; Higher education; Institutional evaluation; No neutrality.

1 El texto es producto de la investigación en desarrollo: "Sistemas de evaluación institucional para universidades públicas, fundamentos conceptuales y estructura de su funcionamiento, casos: Brasil, Colombia, Argentina e Perú", que se realiza con apoyo financiero de la OEA. 


\section{INTRODUCCIÓN}

La evaluación de la calidad de la Educación Superior es un proceso en el que las acciones giran en torno a conceptos fundamentales que sin embargo son polisémicos, como son evaluación y calidad.

Los gobiernos en nombre de la calidad de la educación dictan políticas, los modelos de evaluación de instituciones y programas de educación superior en los diferentes países unos más que otros hacen referencia a la calidad. Se realizan convenciones, congresos internacionales, donde surgen propuestas para promover la calidad de la educación. Se construyen declaraciones en conferencias internacionales promovidas por organismos como las Naciones Unidas donde se establecen compromisos que obligan a las naciones a promover la calidad de la educación.

El concepto de calidad, es central en una serie de exigencias y propuestas, sin embargo los actores con el término calidad frecuentemente no se refieren a los mismos aspectos. La Comisión Nacional de Evaluación y Acreditación Universitaria (CONEAU) de Argentina señala que: "En la medida en que el objetivo de la CONEAU es la evaluación institucional para el mejoramiento de la calidad de las universidades, además de pensar sobre su propio concepto de calidad, debe relacionarlo con los de las universidades" (CONEAU, 1997, p. 6), expresando la existencia de diferentes conceptos de calidad en el mismo ámbito de la educación superior.

El presente artículo busca contribuir a la reflexión sobre la no neutralidad existente en la evaluación de la calidad de la Educación y como esta no neutralidad se manifiesta en los modelos de evaluación de la Educación Superior, haciendo referencia a los casos de Colombia, México, Argentina y Brasil.

\section{CALIDAD Y NO NEUTRALIDAD}

Aun cuando la idea de que algo es mejor que es la semilla del concepto de calidad, ya se manifestó en las primeras elecciones que hizo el hombre en los primeros años de su historia. El concepto de calidad se desarrolló en el mundo empresarial hasta convertirse en la actualidad en una necesidad estratégica y en un arma para sobrevivir en mercados altamente competitivos. Sin embargo ya en el mercado no todos entienden lo mismo cuando se refieren a la calidad. Para el cliente está relacionada a la satisfacción por la utilidad que experimenta con el producto o servicio, para el proveedor está expresada en términos de la efectividad y ventajas de lo que oferta para un uso anunciado, para el empresario es importante el costo-beneficio asociado al producto o servicio. Por lo tanto 
en este ámbito se puede inferir que la característica requerida como referente de calidad está en función al propósito del sujeto que realiza la evaluación de la calidad. En este caso particular, los propósitos del cliente, del proveedor y del empresario, no son los mismos, y cada propósito determina cual es el referente de calidad.

El término de calidad fue tomado del mundo empresarial y utilizado en la educación, generándose también en este ámbito una multiplicidad de conceptos sobre calidad los cuales se pueden encontrar en la literatura especializada, la variedad es tal que es posible establecer tipologias e identificar líneas de análisis.

Morosini (2001) explica el uso del término calidad desde distintos puntos de vista: Calidad sinónimo de evaluación, calidad sinónimo de empleabilidad, calidad sinónimo de respeto a las especifidades, calidad sinónimo de equidad.

Los diferentes puntos de vista mencionados se diferencian por que priorizan un referente de calidad distinto. Cada punto de vista expresa una concepción de calidad y en cada caso son los propósitos de las instituciones, de los programas o de los proyectos, los que determinan los referentes de calidad a utilizar. Sin embargo los propósitos son definidos por el marco ideológico de los promotores.

Orozco (2002, p. 1), manifiesta que "una de las dificultades para formarse una idea de la calidad en sentido estricto y referida específicamente a las universidades es la multiplicidad de acepciones de este término que encontramos en la literatura especializada". Afirma también que los conceptos pueden diferenciarse en función al énfasis en determinadas características que orientan el juicio de calidad sobre el objeto evaluado, por ejemplo: eficiencia, productividad o eficacia. Sin embargo el juicio de calidad fundada en la eficiencia o fundada en la eficacia, estará en función al interés del sujeto que evalúa por que se consiga un propósito considerado valioso. Por ejemplo eficaz para lograr mayor empleabilidad o eficaz para lograr mayor equidad. Luego la no neutralidad de la evaluación de la calidad de la educación se hace evidente en el propósito (académico, social o político) que es de interés de evaluador, en función al cual luego se diseñan y aplican los procedimientos, criterios e instrumentos.

\section{DIMENSIONES DE LA CALIDAD}

No obstante la variedad de puntos de vista que se derivan de la no neutralidad del concepto, es posible proponer un modelo para las dimensiones del concepto calidad.

La calidad primero plantea el problema de la pertinencia de lo que se evalúa para la función o el propósito que se va a cumplir, es decir se dice que el algo 
que se somete a juicio de evaluación, es de calidad cuando tiene características que informan que es útil para cumplir con una determinada función. Si lo que se evalúa es el funcionamiento de una institución, la pertinencia está referida a la utilidad de lo que hace la institución para satisfacer demandas externas.

Luego una primera dimensión de la calidad es la pertinencia. A este respecto dice Orozco (2002, p. 2), "cuando hablamos de calidad en el sentido de la capacidad que tiene una Institución o un sistema de educación superior de satisfacer las demandas externas, estamos realmente refiriéndonos a pertinencia, la cual puede ser una dimensión de la calidad y nada más".

En segundo lugar si lo que se evalúa es pertinente, se plantea el problema de determinar en que medida el algo evaluado, permite el logro de los propósitos previstos. La comparación entre el desempeño esperado con el desempeño real, permite decidir sobre la mayor o menor calidad del algo evaluado. Es así que la eficiencia en el desempeño al constituirse en un criterio diferenciador de la calidad, es otra dimensión de la calidad.

Por otra parte se plantea el problema de la mayor o menor utilidad del algo evaluado para conseguir un resultado deseado que se manifiesta en una modificación de la realidad externa o del pensamiento. Entonces se decide sobre la mayor o menor calidad en función a esa utilidad del algo evaluado para conseguir un impacto deseado, es decir en función a su eficacia. De este modo la eficacia resulta ser otra dimensión de la calidad y el impacto una subdimensión de la eficacia.

Otra exigencia que se formula el juicio de calidad, es sobre las características del desempeño dinámico del algo evaluado, se formulan por ejemplo apreciaciones del plazo en que se logran resultados. $\mathrm{O}$ de frecuencias observadas en el desempeño, por ejemplo número de aciertos, número de errores, etc. Estos conceptos están relacionados a la productividad del desempeño y son también utilizados como criterio diferenciador de calidad. Tiene más calidad si el algo evaluado permite obtener resultados esperados en menor tiempo. Tiene más calidad si el algo evaluado muestra desempeños con menor número de errores. Por ejemplo, la calidad total esta asociada a cero errores. La productividad resulta así otra dimensión de la calidad.

El juicio de calidad que es formulado por un evaluador en base a la pertinencia, eficiencia, eficacia y productividad, puede ser parcial pues la formulación de los referentes puede estar sesgado subjetivamente, por esta razón el juicio de pares externos es importante para dar validez a la evaluación de calidad realizada. Luego la acreditación externa de la calidad resulta ser otra dimensión de la calidad. Pues un sistema cuya calidad ha sido testada por juicio externo, tiene mayor garantía de calidad. 
Por otro lado la calidad establecida en función a eficiencia, eficacia, productividad y acreditación, es afectada por las dinámicas externas al sistema evaluado: de los mercados, el avance de la ciencia y tecnología, el crecimiento del conocimiento, o por dinámicas internas del propio sistema, que hacen que se formulen nuevas alternativas, nuevas propuestas, de manera que el juicio de calidad pierde vigencia, está afectado por la obsolescencia. Luego otra dimensión de la calidad es el aseguramiento de la calidad. Tendrá mayor calidad el sistema que posea mecanismos de aseguramiento de la calidad. Sin embargo la mejora continua de la calidad no se realiza sin la apropiación o generación y aplicación de nuevo conocimiento, por ello la mejora continua de la calidad se sustenta en el aprendizaje continuo del sistema sobre el cual se evalúa la calidad.

El juicio de calidad que hace énfasis en la pertinencia, eficiencia, eficacia, productividad, en el aseguramiento, es aun insuficiente. Para establecer la calidad de un sistema es preciso verificar si el sistema cumple con sus propósitos sin consecuencias internas o consecuencias externas. En este caso se formula por ejemplo el juicio de calidad sobre un sistema cuya dinámica compromete su propia subsistencia o la subsistencia de su entorno. Luego tendrá mayor calidad el sistema que sea más sustentable. Es así que la sustentabilidad se presenta como otra dimensión de la calidad. En la figura 1. se presenta gráficamente la calidad y sus dimensiones.

Figura 1 - Dimensiones de la calidad

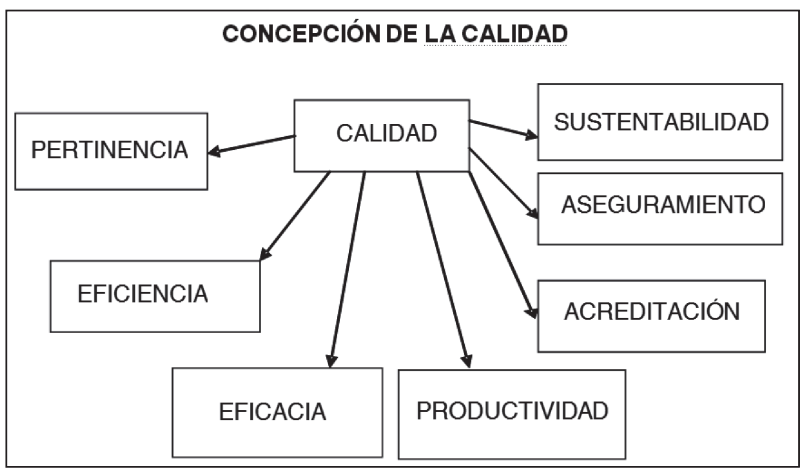

El modelo de las dimensiones de la calidad, puede ser aplicado a casos particulares, por ejemplo, la Oficina Regional de Educación de la UNESCO para América Latina y el Caribe partiendo de que la educación es "un derecho fundamental y un bien público irrenunciable" (OREAL/UNESCO, 2008, p. 6), y tiene como misión el desarrollo integral de ciudadanos de manera que 
sean capaces de transformar la sociedad actual haciéndola mas justa, inclusiva y democrática, propone que la calidad de la educación es "un medio para que el ser humano se desarrolle plenamente como tal, ya que gracias a ella crece y se fortalece como persona y como especie que contribuye al desarrollo de la sociedad, transmitiendo y compartiendo sus valores y su cultura" (p. 7), plantea 5 dimensiones para el concepto de calidad:

\section{1) Equidad, 2) Relevancia, 3) Pertinencia, 4) Eficacia y 5) Eficiencia.}

De acuerdo al modelo propuesto en el presente trabajo, una dimensión que la calidad de la educación es la eficacia, y tiene como una subdimension a la equidad, pues una educación de calidad que da igualdad de oportunidades para todos, es eficaz para todos, es decir es educación de calidad con eficacia para conseguir equidad, luego garantiza que todos los estudiantes de acuerdo a sus capacidades, alcancen los máximos niveles de desarrollo y aprendizaje posibles. Otra subdimensión de la educación de calidad con eficacia, es el impacto social, cuando la educación de calidad es útil para conseguir participación en el desarrollo social, o para desarrollo una cultura de paz, o para desarrollar y transferir a la sociedad tecnologías sociales, o tecnologías renovables. A este respecto la participación en el desarrollo social, el desarrollo de una cultura de paz, y la transferencia de tecnologías sociales, serían subdimensiones del impacto social de una educación de calidad con eficacia.

Por otra parte en el modelo propuesto la relevancia, es considerada una subdimensión de la pertinencia, puesto que la relevancia exige la existencia previa de la pertinencia. El Centro Interamericano para el Desarrollo del Conocimiento en la Formación Profesional (CINTERFOR/OIT, 2006), diferencia dos dimensiones en materia de pertinencia, una de ellas hace referencia a como la formación profesional responde de manera eficaz, eficiente y oportuna a los problemas, demandas y necesidades de dicho entorno, favoreciendo además la vinculación de sus sujetos de atención con él. Es decir una dimensión de la pertinencia hace referencia al entorno. La otra dimensión de la pertinencia hace referencia a las características, condicionamientos, necesidades y expectativas de sus sujetos de atención, y en cómo se toman en cuenta al momento de realizar diseños curriculares, elaborar materiales didácticos, adaptar los enfoques pedagógicos y adecuar el propio funcionamiento y formas de gestión de las instituciones (relevancia). La otra dimensión de la pertinencia hace referencia a los sujetos que son atendidos por la institución. Así la formación profesional es pertinente con el entorno y con la población destinataria. 


\section{DEMANDAS DE CALIDAD COMO FACTORES QUE CONTRIBUYEN A LA NO NEUTRALIDAD DEL CONCEPTO: CASO DE LAS UNIVERSIDADES}

Al ser la concepción de calidad formulada por un actor social, tiene ya de origen un contenido ideológico, pues es el sujeto quien en base a su concepción del mundo da contenido significativo a los elementos de referencia que le permiten emitir el juicio de calidad.

Águila Cabrera (2005, p. 2) afirma que la calidad es un concepto multidimencional sujeto a diversas interpretaciones, y ante la existencia de muchas definiciones de calidad en la literatura actual, es necesario:

determinar aquella que más convenga a la evaluación en las condiciones de la realidad latinoamericana, sin olvidar que la calidad tiene que estar conjugada con la pertinencia y el impacto, pues no se puede concebir una institución universitaria de calidad que no sea pertinente en su entorno social.

Al formular esta concepción parte de una percepción de la realidad latinoamericana, y plantea la necesidad de elegir la definición de calidad que más convenga a un propósito que es valioso para un determinado contexto social, identifica una relación de dependencia entre la calidad de la institución universitaria y demandas de su entorno social que se expresa en términos de pertinencia e impacto.

Águila Cabrera (2005, p. 2) sostiene también que "el concepto de calidad de la educación universitaria cambia de contenido en cada época, el contenido no es estable y duradero porque es un concepto primordialmente histórico", que se modifica con el cambio en las exigencias que plantea la sociedad a la universidad, con la adopción de políticas por los estados como respuesta a situaciones que trajo la globalización, a la explosión indiscriminada de programas e instituciones, al debilitamiento de lo que se entendía por calidad.

Por consiguiente distingue como factores que modifican el contenido del concepto calidad y en consecuencia contribuyen a su no neutralidad, las demandas planteadas desde la sociedad y por los estados hacia las universidades. Estas demandas son diferentes en diferentes épocas de la historia, por lo que en respuesta a ellos el concepto de calidad varía en las diferentes épocas de la historia.

Las demandas de calidad que se plantean a la universidad tienen diversos orígenes, los cuales son mencionados con mayor o menor claridad en la literatura, a partir de diversas fuentes se identifican las siguientes: 


\subsection{Las demandas del Estado}

Que se formulan como exigencias de calidad en el cumplimiento de funciones a la universidad, además de exigencias de cumplimiento de políticas públicas y también aun cuando son los partidos políticos quienes pueblan el aparato estatal con sus partidarios cuando acceden al gobierno, el estado requiere de profesionales y ciudadanos con competencias y capacidades para el buen funcionamiento de órganos de sus instituciones.

Las demandas del estado se expresan en normas jurídicas que buscan implementar políticas públicas, programas y acciones que aseguren nuevas sistemáticas de regulación y gestión en general de la educación superior, y que incluyen temas relativos al financiamiento, autonomía, expansión, calidad, democratización de acceso entre otras.

\subsection{Las demandas sociales}

Que se expresan en exigencias de calidad en la formación profesional y en la oferta de opciones profesionales. Demandas por ampliación del acceso a estudios superiores, por una educación con equidad, para todos con respeto a las diferencias, por formación continuada, actualización y perfeccionamiento, por programas y acciones de preservación y desarrollo de la cultura, por la formación de profesionales y ciudadanos con competencias y capacidades para el desarrollo social, para la incubación y transferencia de alternativas de implementación de tecnologías sociales.

\subsubsection{Acceso y Equidad}

América Latina se caracteriza por tener sociedades con muchas desigualdades debido al modelo de acumulación de la riqueza que beneficia a pequeños sectores de su población, con persistencia de la pobreza, lo cual genera altos índices de exclusión. Esta realidad es claramente observable en los porcentajes de la población entre 25 a 34 años de edad que ha alcanzado educación superior: México 18\%, Brasil 8\%, Chile 18\% (ESPAÑA, 2007).

Las sociedades en consecuencia demandan por acceso a la educación universitaria y que está contribuya a superar las desigualdades de origen de los alumnos (diferentes contextos y culturas, con diferentes capacidades e intereses) para avanzar hacia sociedades más justas, equitativas y democráticas. De acuerdo a Aponte (2008) La mayor parte de la población estudiantil que accede el nivel superior proviene de los Quintiles más altos de ingreso familiar. Luego 
se exige que la educación de calidad deba asegurar el desarrollo y aprendizaje de todos en base al principio de igualdad de oportunidades (dar más a quién más lo necesita y proporcionar a cada quien lo que requiere, en función de sus características y necesidades individuales).

Blanco Guijarro (2006, p. 9) sóstiene que, "una educación no puede ser de calidad si no logra que todos los alumnos, y no sólo parte de ellos, adquieran las competencias necesarias para insertarse activamente en la sociedad y desarrollar su proyecto de vida en relación con los otros. Es decir no puede haber calidad sin equidad". Sin embargo esta demanda debe ir acompañada de políticas económicas y sociales que aborden los factores que generan desigualdad y exclusión fuera del ámbito educativo.

\subsubsection{Formación de ciudadanía}

Otra demanda de la sociedad hacia la Universidad es que además de formar profesionales, que dominen conocimientos propios de sus especialidades, posean también una formación deontológica relativa al ejercicio de las profesiones y una formación humana que contribuya a un comportamiento ético como integrante de la sociedad. Martínez (2006, p. 89), afirma que: "una universidad de calidad y de servicio público es aquella que hace más digna la sociedad, convirtiendo a sus estudiantes tanto en excelentes profesionales, como en ciudadanos y ciudadanas cada vez más cultos y críticos". Plantea por consiguiente que no es adecuado separar la formación profesional de la formación ciudadana. Los aprendizajes que se podrían contribuir a desarrollar ciudadanía, son abordados también por el Informe de la Comisión Delors, al referirse a "aprender a ser", que hace referencia a la construcción de identidad, autonomía y del propio proyecto de vida, y a "aprender a vivir juntos" que implica el respeto a valores del pluralismo, la comprensión mutua y la paz (UNESCO, 1996).

\subsubsection{Calidad y retención estudiantil}

El abandono de los estudios universitarios es otra problemática de interés de la sociedad y que aqueja a las instituciones universitarias, las cuales implementan estrategia de retención de la población estudiantil. Los objetivos centrales de los programas de retención son facilitar el acceso de los estudiantes a la educación universitaria, promover su permanencia en las aulas y acompañar sus procesos para la culminación de los planes de estudio en los tiempos propuestos para ello. 
La educación universitaria por lo tanto es de calidad si es eficaz para conseguir la retención de los estudiantes en las aulas.

\subsubsection{Otras demandas de la sociedad}

En el caso de Brasil algunas de las demandas de la sociedad para con la educación se han recogido en la actual Constitución de 1988 que en su articulo 214 determina que se establezca un Plan Nacional de Educación plurianual, condujese a la erradicación del analfabetismo, a la universalización de la atención escolar, a la mejora la calidad de la enseñanza, a la formación para el trabajo, y a la promoción humanística, científica y tecnológica del País.

\subsection{Las demandas del sector empresarial}

Se manifiestan como exigencias de calidad en la formación de capital humano con competencias y capacidades para el desarrollo empresarial, para el desarrollo de tecnologías de la producción de bienes y servicios. Demandas por aprendizajes y desarrollo de conocimiento específicos.

En el informe final del proyecto TUNING - LATINOAMERICA 20042007, se señala las competencias más y menos importantes mencionadas por los emperadores en América Latina, luego de una encuesta realizada a 1669 empleadores, los resultados se muestran en la tabla $\mathrm{N}^{\mathrm{o}} 2$.

Tabla 1 - Las seis competencias más y menos importantes según los empleadores en América Latina

\begin{tabular}{l|l}
\hline \multicolumn{1}{c|}{ COMPETENCIAS MÁS IMPORTANTES } & COMPETENCIAS MENOS IMPORTANTES \\
\hline Compromiso ético & $\begin{array}{l}\text { Valoración y respeto por la diversidad y } \\
\text { multiculturalidad }\end{array}$ \\
\hline Compromiso con la calidad & Capacidad de investigación \\
\hline Capacidad de aprender y actualizarse & Compromiso con su medio sociocultural \\
\hline $\begin{array}{l}\text { Capacidad de aplicar conocimientos en la } \\
\text { práctica. }\end{array}$ & $\begin{array}{l}\text { Compromiso con la preservación del medio } \\
\text { ambiente }\end{array}$ \\
\hline $\begin{array}{l}\text { Capacidad para identificar plantear y } \\
\text { resolver problemas }\end{array}$ & $\begin{array}{l}\text { Habilidad para trabajar en contextos } \\
\text { internacionales }\end{array}$ \\
\hline Capacidad de trabajo en equipo & $\begin{array}{l}\text { Capacidad de comunicación en un segundo } \\
\text { idioma. }\end{array}$ \\
\hline
\end{tabular}

Fuente: Pablo Beneitone et al. 2007. Reflexiones y perspectivas de la Educación Superior en América Latina. Proyecto Tuning América Latina 2004-2007, p. 60. 
Se observa que los empleadores consideran como competencias menos importantes las que están relacionadas más próximamente a las demandas de la sociedad, tales como el compromiso con su medio sociocultural, con la preservación del medio ambiente, con la valoración y respeto por la diversidad y multiculturalidad. Por lo tanto se ratifica que las exigencias de calidad que plantea el sector de los empleadores, tiene diferentes referentes, por lo tanto el juicio de calidad que emita este sector sobre las instituciones y programas no expresará el interés de la sociedad.

\subsection{Las demandas de desarrollo de Ciencia y tecnología}

Generadas por el crecimiento explosivo de la información académica y del conocimiento, que se expresan en necesidades de investigación y desarrollo, exigencias por la formación de investigadores con las competencias y capacidades para la generación del conocimiento.

Desde este punto de vista el papel de la educación universitaria en estos tiempos es el de contribuir con el desarrollo de la sociedad del conocimiento y de las competencias y los servicios que se necesitan para la creación de conocimiento y su utilización en la producción de bienes y servicios.

\subsection{Demandas de la universidad}

A su vez la universidad plantea al Estado entre otras, exigencias de autonomía, financiamiento, estabilidad de normas educacionales, mejores salarios para la docencia, y a la sociedad requisitos de formación académica para los ingresantes.

Es claro que dependiendo de la fuente de exigencia de calidad hacia la universidad, el concepto de calidad no es el mismo, aun entre instituciones universitarias, y entre modelos de evaluación de la educación superior, los indicadores de calidad no son los mismos, del mismo modo los indicadores que expresan la calidad de las actividades universitarias requeridas para producir ciencia y tecnología, no son los mismos que los indicadores que expresan la calidad de los profesionales universitarios que es valorada por la empresa. Por otro lado para la sociedad una universidad es de calidad cuando contribuye al desarrollo social, cuando su quehacer tiene impacto social, en tanto que para el sector empresarial una universidad es de calidad si sus profesionales contribuyen a la generación de utilidades para el desarrollo empresarial.

Los indicadores expresan el contenido del concepto calidad para los distintos demandantes de calidad y varían en cada concepto, porque cada demandante tiene sus propios propósitos y elige los indicadores que considera pertinentes. 
Figura 2 - Demandas como factores que modifican el contenido del concepto calidad

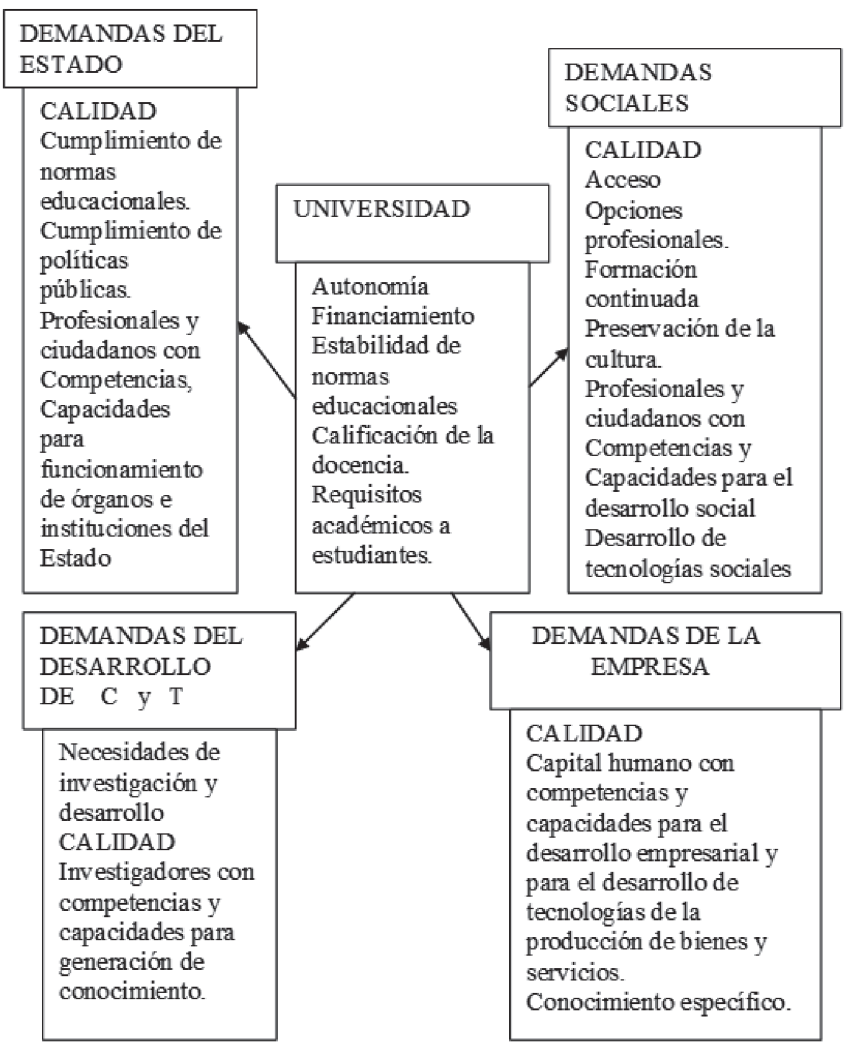

El propósito del demandante por consiguiente es importante para establecer el contenido del concepto calidad. Esto queda muy claro con el siguiente ejemplo: la CAPES como demandante tiene como uno de sus propósitos evaluar la calidad en los post-grados, el contenido de la calidad expresado por sus indicadores, es diferente al concepto de calidad que tiene el CONAES, que tiene como propósito evaluar la calidad de instituciones y programas de graduación. Ambos organismos tienen diferentes propósitos y utilizan diferentes contenidos para el concepto calidad, para CAPES son importantes entre otros indicadores los de resultados (producción bibliográfica, publicaciones, producción técnica, tesis y disertaciones), para CONAES además de los de resultado obtenidos por aplicación del ENADE, son importantes: la intención de la institución que 
se expresa en la propuesta institucional contenida en el proyecto académico $\mathrm{y}$ en el proyecto institucional, los indicadores de insumo que se utilizan en la evaluación institucional y la implementación de mejoras como producto de la autoevaluación y la evaluación externa.

\section{LA NO NEUTRALIDAD EN LOS MODELOS DE EVALUACIÓN DE LA EDUCACIÓN SUPERIOR, CASOS DE: COLOMBIA, MÉXICO, ARGENTINA Y BRASIL}

El modelo de referencia (elementos de referencia considerados valiosos para el evaluador) utilizado para la evaluación institucional y de programas, constituye la propuesta ideológica particular que expresa la no neutralidad existente en la construcción de cada uno de estos modelos de evaluación y como característica diferencial permite identificar diferentes implicancias en su funcionamiento.

\subsection{Caso colombiano}

En el caso colombiano el Consejo Nacional de Acreditación (CNA) es el órgano del Sistema Nacional de Acreditación, que preside, organiza, fiscaliza, da fe de su calidad y finalmente recomienda al Ministro de Educación Nacional acreditar programas e instituciones que lo merezcan y señala metas de crecimiento a las que todavía no llegan según niveles de calidad convenidos. El Consejo Nacional de Educación Superior (CESU) por su parte, tiene atribuciones para señalar políticas que permitan organizar y poner en marcha el Sistema Nacional de acreditación, de acuerdo a este organismo,

La acreditación es el acto por el cual el Estado adopta y hace público el reconocimiento que los pares académicos hacen de la comprobación que efectúa una institución sobre la calidad de sus programas académicos y funcionamiento y el cumplimiento de su función social (COLOMBIA, 1995, p. 2).

El sistema de acreditación busca preservar el carácter voluntario, mantener la naturaleza eminentemente académica del proceso evaluativo, operar en forma tal que goce de credibilidad y mantener niveles de calidad reconocidos internacionalmente.

En el procedimiento para la acreditación de programas, la autoevaluación es realizada por las instituciones de Educación Superior (IES) siguiendo los lineamientos (factores, características de calidad, aspectos que se deben evaluar, indicadores), metodología, criterios, e instrumentos establecidos por el CNA. 
Luego de la autoevaluación, la evaluación por pares y la evaluación final por el CNA, el acto de acreditación es promulgado por el Ministro de Educación.

Para obtener la acreditación de programas se requiere del cumplimiento de parámetros (estándares de calidad) previamente definidos por el Consejo Nacional de Acreditación con la colaboración de la comunidad académica. Es así que se establecen estándares de calidad para programas, se exigen condiciones mínimas de calidad para creación y funcionamiento y características de alta calidad para los programas que quieren ser reconocidos por su excelencia en un proceso de acreditación. "Un estándar de calidad puede ser entendido como un patrón, una medida esperada o un indicador de desempeño que debe ser alcanzado para legitimar un programa académico" (ROA, 2003, p. 9).

La acreditación tiene carácter temporal y es el resultado del cumplimiento de exigencias y de la acumulación de requisitos necesarios para la buena calidad académica de la institución y de sus programas.

\subsubsection{Apreciación}

Este modelo es respetuoso del mandato constitucional por el cual se establece que la educación en Colombia es un servicio público, responsabilidad del Estado, la sociedad y la familia, y que corresponde al Estado "velar por su calidad, por el cumplimiento de sus fines y por la mejor formación moral, intelectual y física de los educandos" (Colombia.2009). Además asigna un alto valor al rol de la Educación Superior en desarrollo social, cuando reconoce que la Educación Superior tiene una función social estratégica y que por tanto debe ser prestada con la mayor calidad posible (COLOMBIA, 2006).

El énfasis en la responsabilidad estatal sobre la calidad del servicio público, conduce a la construcción del modelo de referencia en base a estándares de calidad, los cuales son establecidos por el órgano estatal con la contribución de la comunidad académica y se utilizan para evaluar los niveles de calidad de los programas o instituciones, es así que el modelo de referencia en la propuesta permite asignar una alta calidad o una calidad mínima a los programas o instituciones evaluados.

El estándar de calidad, es tomado como patrón de comparación, y se hace referencia al reconocimiento internacional del nivel de calidad que es informado por estos patrones.

Sin embargo la existencia de un nivel de calidad de validez internacional, entra en contradicción con el reconocimiento del cumplimiento de función 
social de la institución que se hace en el acto de acreditación. Por cuanto la función social de una institución es valorada prioritariamente por la sociedad a la cual ella pertenece. Es la comunidad de su entorno más próximo la que experimenta más directamente la contribución de la institución. En este sentido, la función social es valorable en relación a una determinada sociedad ya que se refiere no solo a la transferencia de tecnología, sino también a la preservación de su patrimonio cultural, a la construcción de los Estados, al desarrollo de formas de organización social, etc. La calidad de la función social que cumple una universidad en Colombia no es comparable con la calidad de la función social que cumple otra universidad en la Patagonia Austral de Argentina o en el nordeste de Brasil.

Por ello el nivel de calidad de una institución o programa desde el punto de vista de la función social que cumple para su sociedad, no puede ser comparable internacionalmente.

Cuando se postulan niveles de calidad reconocidos internacionalmente, se informa de la determinación de la calidad de instituciones y programas en base a la utilización de estándares internacionales, que reducen los niveles de calidad a los esperados por la comunidad internacional y se soslaya la importancia de la función social de la universidad, ya que esta no es valorable con estándares internacionales. En otras palabras se postula la formación de egresados de programas e instituciones para el mercado global.

\subsection{Caso argentino}

En el caso argentino la Comisión Nacional de Evaluación y Acreditación Universitaria (CONEAU) es el organismo que tiene como objetivo la evaluación institucional para el mejoramiento de la calidad de las universidades. El documento orientador emitido contiene los Lineamientos para Evaluación Institucional (CONEAU, 1997) en los que se hace referencia a las variables básicas que serán objeto de análisis.

La CONEAU considera que la evaluación institucional contempla dos fases: la autoevaluación y la evaluación externa, cada una de ellas con diferentes actores.

El proceso de evaluación institucional tiene como premisa básica el respeto irrestricto a la autonomía universitaria, principio que goza de jerarquía constitucional (art. 75. inc. 19 de la Constitución de la Nación Argentina) y al proyecto institucional que cada institución determine para sí misma. 
La concepción de evaluación en la CONEAU, es que la evaluación institucional "supone un proceso complejo para determinar el valor de algo, que implica una delicada tarea de interpretación de un conjunto de elementos que interactúan configurando una realidad particular y significativa" (CONEAU, 1997, p. 6).

A partir de este concepto, el modelo de referencia es construido en el diálogo, la discusión y la reflexión sobre acciones y realizaciones de las instituciones evaluadas, y toma la forma del proyecto institucional, el cual da importancia a las particularidades propias de cada institución. Pérez Rasetti (2009, p. 12) al referirse a los Lineamientos para Evaluación Institucional, afirma que "para la CONEAU, en razón de la autonomía, el modelo contra el cual debe realizarse la evaluación institucional de cada universidad es su propio proyecto institucional".

\subsubsection{Apreciación}

La evaluación institucional en la Argentina, en sus inicios tuvo poca aceptación por parte de la comunidad universitaria, por lo que su implementación fue en las universidades que voluntariamente se integraron al proceso, teniendo como garantías, el respeto a la autonomía y a su proyecto institucional. En los lineamientos sólo se hace una referencia ligera a las variables sobre las cuales deberá centrarse el análisis en cada institución, sin precisar criterios e indicadores de evaluación.

En la autoevaluación la institución tomando como referencia los lineamientos propuestos por la CONEAU construye su proyecto institucional (referente para evaluación) la estructura y metodología para la elaboración del proyecto, es establecida libremente por cada institución, los lineamientos sólo proporcionan variables de análisis a ser tomadas en cuenta en la reflexión colectiva, la misión, visión, objetivos estratégicos, metas, programas, actividades, fuentes de verificación; son formulados también libremente. Lo que se consigue con este proceder es promover espacios para la reflexión colectiva y un mejor conocimiento de las debilidades y fortalezas en el funcionamiento de la institución por la comunidad educativa. Inicialmente si existe identificación con la propuesta de cambio, se pueden plantear mejoras que no requieran recursos adicionales y que se reducen a actividades que tienen como eje la interacción docente - alumno. En el caso de universidades estatales, el modelo desde esta etapa plantea restricciones de orden financiero para la propuesta de mejoras. Así la mejora de las instituciones se apoya en las mejoras viables desde el punto de vista de los recursos. 
Para la etapa de evaluación por pares externos las deficiencias encontradas deberán haber merecido la aplicación de políticas de mejoramiento institucional con las asignaciones presupuestales necesarias. En esta etapa deberá hacerse una distinción entre las medidas de mejoramiento que serán posibles de realizar con el presupuesto disponible y las medidas que requerirán de presupuesto adicional.

Sin embargo existirán mejoras que requerirán financiamiento que puede ser temporal, por ejemplo la implementación de laboratorios, otras requerirán ampliación presupuestal permanente, por ejemplo contar con un mayor número de docentes a tiempo completo o dedicación exclusiva, requeridos para resolver el problema de la masificación estudiantil generada por el ingreso universal (problema importante para la universidad argentina), a fin de mejorar la calidad del servicio educativo así como la realización de actividades de investigación, que se constituyen en deficiencias cuando se tiene insuficiente número de docentes, o mayor número de docentes a tiempo parcial. En el caso de universidades nacionales que se mantienen con recursos estatales, en tanto no se den estas ampliaciones presupuestales este tipo de problemas no será resuelto.

En cuanto al referente utilizado para la evaluación, el proyecto institucional para contribuir al mejoramiento institucional, debería ser hecho utilizando metodologías de planificación estratégica o de marco lógico, con especificación de la previsión financiera para programas y actividades de mejoramiento a realizar. En los casos revisados, las acciones en los planes institucionales no están acompañadas de la previsión financiera.

El resultado de la evaluación externa es un informe final con recomendaciones que son de carácter público y se espera que se produzcan cambios y mejoras a lo largo del tiempo.

En el supuesto de la existencia de recursos financieros, la limitación de este modelo es que la mejora institucional depende en gran medida de la gestión y liderazgo de las autoridades, de la búsqueda de consensos, de la negociación política (aspecto importante en universidades politizadas) y de la participación e identificación colectiva de la comunidad académica con el proyecto.

El modelo parte del supuesto teórico de la cooperación diligente de todos los integrantes de la comunidad académica en torno de la construcción e implementación del proyecto institucional, en el marco de una actitud decidida de los gobernantes de turno de incrementar el presupuesto de las universidades nacionales o de promover programas de apoyo financiero. Comportamiento que de darse, puede ser coyuntural; pues la tendencia es que los gobernantes buscan reducir el presupuesto de las universidades públicas, empujando cada vez más a estas instituciones al autosostenimiento. 


\subsection{Caso brasileño}

En el caso brasileño, el Estado regula, fomenta y supervisa el sistema de evaluación en su conjunto, se reconoce el papel estratégico de la Educación Superior, en la concepción del Sistema Nacional de Evaluación de la Educación Superior (SINAES), creado por Lei $n^{\circ} 10.861$, de 14 de abril de 2004, en la cual se establece que el SINAES tiene como una de sus finalidades la mejora de la calidad de la educación superior. Se reconoce la existencia de dos orientaciones fundamentales en la evaluación, una de carácter regulatorio que hace énfasis en obtener información objetiva de resultados y productos, y otra de carácter formativo/emancipatorio que considera importante la presencia de las evaluaciones formativas, participativas, orientadas a los procesos, a la diversidad y complejidad de las instituciones y el fortalecimiento de la función pública de la educación. El Sistema Nacional de Evaluación de la Educación Superior propone articular las dos orientaciones, regulación y evaluación educativa de naturaleza formativa. Tiene como antecedente el Programa de Evaluación Institucional de las Universidades Brasileñas (PAIUB) del año 1993, en el cual se concibió que la autoevaluación fuera la etapa inicial de un proceso, que se completaba con la evaluación externa de la institución.

La Comissão Nacional para a Avaliação da Educação Superior (CONAES), es el órgano colegiado de coordinación y supervisión del SINAES, proporciona las directrices para la autoevaluación institucional, que orientan hacia la realización de un diagnóstico más exhaustivo de la institución. El proceso es responsabilidad de cada institución.

El relatorio de autoevaluación debe contener información de elementos de evaluación, análisis cualitativos e acciones que la IES pretende emprender como resultado del proceso de autoevaluación, identificando los medios y recursos necesarios para la realización de mejoras, así como una evaluación de aciertos y equívocos del propio proceso de evaluación. Es parte importante del material que será empleado en la evaluación externa.

En la etapa de evaluación externa los relatorios deben contener un análisis de los puntos fuertes, de las principales carencias y también de las posibilidades y potencialidades de la institución, así como también recomendaciones explicitas a las instituciones y órganos del Ministerio de Educación y Ciencia responsables por los procesos de regulación y evaluación.

En base a los pareceres de la evaluación externa las instancias competentes del MEC, toman las decisiones sobre autorización, reconocimiento, acreditación, reacreditación, etc. Una vez finalizado el proceso de evaluación, un nuevo ciclo de evaluación se abre para las instituciones, debiendo superar en él, las 
deficiencias verificadas y cumplir las obligaciones y exigencias hechas por el poder público en el ciclo anterior en plazos establecidos (SINAES, 2007).

\subsubsection{Apreciación}

La evaluación de programas e instituciones tiene en el caso brasileño un referente más complejo, se alimenta no solamente de la información generada en el proceso a partir de tres componentes principales: la evaluación de las instituciones comprendiendo la evaluación interna (autoevaluación) y la externa, la evaluación de los programas de graduación (cursos) y los resultados del Examen Nacional de Desempeño de los Estudiantes (ENADE), sino que también de la información existente procedente del Censo de la Educación Superior y del registro de IES.

La evaluación abarca por lo tanto datos cuantitativos y cualitativos. Sin embargo existen datos cuantitativos que sirven más para construir prestigio institucional que para evaluar la calidad de los procesos por cuanto se refieren sólo a resultados. Otro problema es que cuando existe diversidad de instrumentos y diferentes instituciones que participan en la implementación del proceso de evaluación, se hace de manifiesto una debilidad en la consistencia del sistema pues los instrumentos obedecen a distintas concepciones sobre evaluación, no resulta claro qué debería ser más valorado, la evaluación cuantitativa o la evaluación cualitativa.

El éxito en la aplicación del modelo requiere de la participación efectiva y comprometida de los miembros de la comunidad educativa, que solo se consigue si existe una desarrollada "cultura de evaluación" en la institución. La participación requiere además búsqueda de consensos y procesos de negociación. Con limitada participación se fragiliza el impacto de la autoevaluación para la mejora de la institución.

La conformación de la Comissão Própria de Avaliação - CPA, puede requerir de la negociación política para un trabajo efectivo. La participación de los integrantes requiere de una etapa de sensibilización y familiarización previa, donde se motive con la información referente al proceso de evaluación institucional y acreditación.

La conformación de un sistema para evaluación de la Educación Superior (SINAES), en base a dos órganos diferentes, el uno colegiado de coordinación y supervisión del sistema, que es la Comisão Nacional para la Avaliação da Educação Superior (CONAES), creada por Ley 10.861 de 14 de abril de 2004 y otro de naturaleza autónoma que es responsable por la elaboración de instru- 
mentos y la ejecución de los procesos, que es el Instituto Nacional de Estudos e Pesquisas Anisio Teixeria (INEP) creado por a la Ley 378 de 13 de enero de 1937, transformado en autarquía federal vinculada al Ministerio de Educación según lo dispuesto por la Ley 9.448 de 14 de marzo de 1997 modificada por la Ley 10.269 de 29 de agosto de 2001, constituye una debilidad en la estructura organizacional del sistema, por cuanto el INEP con existencia previa, tiene finalidades y atribuciones propias. Mientras el CONAES focaliza su atención en la Educación Superior el INEP se ocupa realizar levantamientos estadísticos y evaluativos en todos los niveles y modalidades de enseñanza.

En el INEP de seis (06) Directorías, una (01) se encarga de la evaluación de la Educación Superior, con lo que se hace de manifiesto que la coordinación no es directa entre los que conciben y los que ejecutan, por respeto al principio de autoridad de las organizaciones.

Esta dificultad para la coordinación se hizo notoria en los resultados del trabajo de investigación realizado por CONAES sobre su imagen pública en las IES brasileñas con una muestra de 532 representantes de las IES, donde preguntados los entrevistados sobre su percepción de los niveles de cooperación en el proceso de SINAES entre a CONAES responsable por la coordinación y supervisión de la evaluación y el INEP, responsable por la elaboración de los instrumentos y la ejecución de los procesos evaluativos. . "Frente a la frase 'El INEP ha ejecutado adecuadamente las directrices de la CONAES en el proceso de evaluación', menos de la mitad de los entrevistados $45.87 \%$ respondió que estaba muy de acuerdo con esta afirmación" (TRINDADE, 2007, p. 86).

Otra debilidad es que la estabilidad de criterios en el modelo de evaluación en las instituciones que implementan el SINAES, como son CONAES, e INEP, es sensible a los cambios de gobernantes, que por una parte traen cambios de responsables de organismos que por lo tanto tienen nuevas concepciones, y por otra modifican los fines de las instituciones. Una muestra de ello es la observación que se hace al Concepto Preliminar de Curso, instituido por la Portarla No 4 de 5 de agosto de 2008 y de la valorización dentro de él, de los resultados del examen muestral ENADE, como resultados que al ser publicados, tienden a sustituir todo el modelo de referencia del SINAES para la evaluación de instituciones y programas, por un conjunto de indicadores que informan sólo sobre resultados de aprendizaje y de manera no confiable, debido a que éstos no forman parte de la evaluación del aprendizaje de los estudiantes que realiza la institución, es decir no forman parte del historial académico del programa que sigue el alumno, por lo que se pone en duda que comprometan su atención, interés o preocupación por los resultados obtenidos. 
La utilización del Concepto Preliminar de Curso difundido en nota técnica por el INEP (2008), como un indicador público y previo de la calidad de los cursos de graduación que combina el desempeño obtenido por los estudiantes en el Examen Nacional de Desempeño de los Estudiantes (ENADE), con los resultados del indicador Diferencia de Desempeño (IDD) y con las informaciones de infraestructura, e instalaciones físicas, recursos didáctico-pedagógicos y cuerpo docente ofrecidas por el curso de una determinada Institución de Enseñanza Superior. Que además permite la elaboración de ranking con los que se clasifica la "calidad" de los cursos de las IES, constituye en la práctica un sistema de evaluación de la Educación Superior paralelo que es utilizado por la comunidad para clasificar los programas y por ende las IES en base sólo a información cuantitativa. La propuesta deja de lado la orientación formativa de la evaluación y se aleja de la señalada por Trindade (2007, p. 13), cuando afirma que: el análisis sistemático e integrado de la información procedente de la evaluación institucional interna y externa, del Examen Nacional de Desempeño de los Estudiantes (ENADE), y de la evaluación de cursos de graduación, permitirá una mejor comprensión del funcionamiento, de las calidades, y de las insuficiencias de cada institución del Sistema de Educación Superior.

\section{CONCLUSIONES}

a) Contribuyen a la no neutralidad de la evaluación de la calidad: el énfasis conceptual en alguna o algunas dimensiones de la calidad, el propósito que es de interés del evaluador, el cual determina el referente de calidad a utilizar, y las demandas de calidad endógenas y exógenas a la institución, programa o sistema que es objeto de evaluación.

b) El referente de la evaluación es una característica esencial de los modelos de evaluación, de manera que diferentes modelos utilizan diferentes referentes. La concepción de calidad está en función al marco ideológico de los evaluadores, por consiguiente, también la concepción del referente de la evaluación de calidad, el cual se configura en los modelos con diferente participación del Estado, de agencias y de integrantes de las instituciones, como respuesta a demandas académicas, sociales y políticas que son diferentes en los diferentes paises.

c) El modelo colombiano es un modelo que exige de las IES cumplir con requisitos de desempeño establecidos por estándares de calidad de validez 
internacional previamente establecidos por el organismo estatal acreditados con la contribución de la comunidad académica, sin embargo es discutible que permitan evaluar adecuadamente la calidad de la función social de la IES.

d) En el modelo argentino se deja a las instituciones construir su modelo de referencia para evaluación, en respeto a la autonomía universitaria y la mejora de las institución esta en función a la gestión, la negociación política, la identificación de los miembros de la comunidad educativa con el proyecto institucional y a los recursos disponibles.

e) En la evaluación de la Educación Superior brasileña el SINAES hace uso de información cuantitativa e información cualitativa, procedentes de la evaluación institucional, la evaluación de programas y el ENADE, sin embargo no resulta clara la consideración final en términos de importancia de los resultados que provienen de la evaluación cuantitativa y de los que proceden de la evaluación cualitativa. En la práctica se producen limitaciones en la coordinación entre CONAES e INEP, y se ha difundido el Concepto Preliminar de Curso como indicador público cuantitativo de la calidad de los programas, que se aparta de orientación formativa y comprensiva del funcionamiento, de las calidades, y de las insuficiencias de cada Institución de Educación Superior.

\section{REFERENCIAS}

ÁGUILA CABRERA, Vistremundo. El concepto de calidad en la educación universitaria: clave para el logro de la competitividad institucional. Revista Iberoamericana de Educación, Madrid, n. 35, mar. 2005. Disponible en: $<$ www.rieoei.org/deloslectores/880Aguila.PDF>. Acceso en: 12 dic. 2009.

APONTE, Eduardo. Desigualdad, Inclusión y Equidad en la Educación Superior en América Latina y el Caribe: Tendencias y escenario alternativo en el horizonte 2021. In: GAZZOLA, Ana Lucia; DIDRIKSSON, Axel (Ed.). Tendencias de la educación superior en América latina y el Caribe. Caracas, Venezuela, IESALC-UNESCO, 2008. cap . 4, p. 21-54. Disponible en: <www.iesalc.unesco.org.ve>. Acceso en: 4 dic. 2009. 
ARGENTINA. Constitución de la Nación Argentina. Buenos Aires, 22 ago. 1994. Disponible en: < http://web.presidencia.gov.co/constitucion/ index.pdf >. Acceso en: 16 dic. 2009.

BLANCO GUIJARRO, Rosa. La equidad y la inclusión social: uno de los desafíos de la educación y la escuela hoy. REICE. Revista Electrónica Iberoamericana sobre Calidad, Eficacia y Cambio en Educación., v. 4, n. 3, p. 1-15. 2006. Disponible en : <http://redalyc.uaemex.mx/redalyc/src/inicio/ ArtPdfRed.jsp?iCve=55140302>. Acceso en: 20 nov. 2009.

BENEITONE, Pablo et al. Reflexiones y perspectivas de la Educación Superior en América Latina. Proyecto Tuning. América Latina 2004-2007. Universidad de Deusto. 2007. 427 p.

BRASIL. Lei $\mathrm{n}^{\mathrm{o}} 10.861$, de 14 de abril de 2004. Instituye el Sistema Nacional de Evaluación de la Educación Superior (SINAES), la Comisión Nacional de Evaluación de la Educación Superior (CONAES) y, señala que evaluación de instituciones, cursos y desempeño de estudiantes será responsabilidad del INEP. Diario Oficial da União, Brasilia, DF, 15 abr. 2004, p. 3. Disponible en:< http://portal.mec.gov.br/arquivos/pdf/leisinaes. pdf $>$ Acceso en: 7 feb. 2010.

BRASIL. Lei n ${ }^{\circ} 9.448$, de 14 de março de 1997. Transforma o Instituto Nacional de Estudos e Pesquisas Educacionais- INEP em Autarquia Federal e estabelece finalidades. Diario Oficial da União, Brasilia, DF, 15 mar. 1997. Disponible en: < http://www.planalto.gov.br/ccivil_03/Leis/L9448. htm>. Acceso en: 7 feb. 2010.

BRASIL. Portaria Normativa $\mathrm{n}^{\circ}$ 4, de 5 de Agosto de 2008. Regulamenta a aplicação do conceito preliminar de cursos superiores, no ámbito do ciclo avaliativo do SINAES. Diario Oficial da União, Brasilia, DF, 7 ago. 2008, Seção 1, p. 15. Disponible en: <http://www.ufpb.br/avalies/ PORTARIA \%20NORMATIVA4.htm> Acceso en: 8 feb. 2010.

CINTERFOR/OIT. Calidad, pertinencia y equidad. Un enfoque integrado de la formación profesional. Montevideo, 2006. Disponible en: $<$ http:// www.cinterfor.org.uy/public/spanish/region/ampro/cinterfor/publ/caper_eq/ index.htm>. Acceso en: 19 nov. 2009. 
COLOMBIA. Acuerdo No 06 de 14 de Diciembre de 1995. Establece políticas del Sistema Nacional de Acreditación. Consejo Nacional de Educación Superior (CESU). Santafé de Bogotá D.C., 14 dic. 1995. Disponible en: < http:/www.cna.gov.co/1741/articles-186370_acuerdo_ cesu_0695.pdf $>$. Acceso en: 6 mar. 2010.

COLOMBIA. Constitución Política. Bogotá, D.E., julio 6 de 1991. Actualizada en mayo de 2008. Disponible en: < http://web.presidencia.gov. co/constitucion/index.pdf >. Acceso en: 16 dic. 2009.

\section{COLOMBIA.CONSEJO NACIONAL DE ACREDITACIÓN. Sistema}

Nacional de Acreditación en Colombia, 2009. Disponible en: <http:/www. cna.gov.co/1741/article-186365.html>. Acceso en: 7 ene. 2010.

COLOMBIA.CONSEJO NACIONAL DE ACREDITACIÓN. Lineamientos para la acreditación de programas. Bogotá D.C. Colombia, 2006. Disponible en: < http://www.cna.gov.co/1741/articles-186359_lineamientos_2.pdf>. Acceso en: 15 ene. 2010.

CONEAU - Comisión Nacional de Evaluación y Acreditación Universitaria. Lineamientos para la evaluación institucional. Resolución $\mathrm{n}^{\boldsymbol{o}}$ 094-CONEAU. Buenos Aires, 1997. Disponible en: <http://www.coneau. edu.ar/archivos/482.pdf>. Acceso en: 1 dic. 2009.

INEP - INSTITUTO NACIONAL DE ESTUDOS E PESQUISAS EDUCACIONAIS ANÍSIO TEXEIRA. Cálculo do conceito preliminar de cursos de graduação. Nota técnica. 2008. Disponible en $<$ http://enade.inep. gov.br/enadeResultado/pdfs/nota_tecnica\%20-\%20CPC.pdf $>$. Acceso en: 10 feb. 2010 .

INEP - INSTITUTO NACIONAL DE ESTUDOS E PESQUISAS EDUCACIONAIS ANÍSIO TEXEIRA. SINAES - SISTEMA NACIONAL DE AVALIAÇÃO DA EDUCAÇÃO SUPERIOR. Da concepção á regulamentação, Brasilia: INEP, 2007. Disponible en: <http://www.paiung. org.br/arquivos/sinaes_4_edicao.pdf>. Acceso en: 25 feb. 2010.

MARTÍNEZ, Miguel. Formación para la ciudadanía y educación superior. Revista Iberoamericana de Educación, OEI, Madrid, n. 42, p. 85-102, 2006. Disponible en: <http://www.rieoei.org/rie42a05.pdf>. Acceso en: 25 nov. 2009. 
MINISTERIO DE EDUCACIÓN Y CIENCIA (MEC). Panorama de la Educación Indicadores de la OCDE 2007. Disponible en: <http://www. mec.es/multimedia/00004656.pdf>. Acceso en: 24 nov. 2009.

MOROSINI, Marília Costa. Calidad de la educación universitaria: isomorfismo, diversidad y equidad. Interface: Comunicação, Saúde e Educação, Botucatu, SP, v. 5, n. 9, p. 89-102, ago. 2001.

OREAL/UNESCO Santiago. Reflexiones en torno a la evaluación de la calidad educativa. Santiago, Chile. Salesianos Impresores S.A., 2008. Disponible en: < http://unesdoc.unesco.org/images/0017/001776/177648S. pdf $>$. Acceso en: 12 nov. 2009.

OROZCO, Luís Enrique. La calidad de la universidad. más allá de toda ambigüedad. 2002. Disponible en: < http://www.cna.gov.co/1741/ articles-186502_doc_academico3.pdf.>. Acceso en: 17 nov. 2009.

PÉREZ RASETTI, Carlos. Cuestiones teóricas y metodológicas de evaluación y acreditación. In: CURSO LATINOAMERICANO DE POLÍTICAS PUBLICAS Y EVALUACIÓN PARA LA EDUCACIÓN SUPERIOR, 2009, Foz de Iguazú. Universidad Federal de la Integración latinoamericana UNILA. Disponible en: <http://ead.pti.org.br/unila/course/ view.php?id=7>. Acceso en: 7 nov. 2009.

ROA, Alberto. Hacia un modelo de aseguramiento de la calidad en la educación superior en Colombia: estándares básicos y acreditación de excelencia. Comisión Nacional de Acreditación de Colombia: 2003. Disponible en: $<\mathrm{http}: / /$ www.cna.gov.co/1741/articles-186502_doc_ academico8.pdf>. Acceso en: 21 mar. 2010.

TRINDADE, Hélgio. Desafios, institucionalização e imagem pública da CONAES. Brasilia: UNESCO/ MEC, 2007.

UNESCO. La educación encierra un tesoro. Informe de la UNESCO de la Comisión Internacional sobre la educación para el siglo XXI. México: UNESCO, 1996. 
